\title{
Value of the chest radiograph before cardiac catheterisation in adults
}

To be of value a diagnostic procedure must be likely to influence the clinical management in an appreciable proportion of the patients to whom it is applied. There are few published reports on the usefulness of a precatheterisation chest radiograph, possibly because it is an unexciting topic. In the current climate of securing value for money, however, we need to reconsider in more detail the role of the precatheterisation chest radiograph.

Areas of concern include the contribution of the radiograph to the assessment of the patient's clinical condition, the performance of the catheterisation procedure itself, the value of the examination in screening for other occult conditions, the radiation burden on the population, and the cost implications-by no means the least important factor.

On page 540 of this issue Stables and TrotmanDickenson concentrate on the extent to which the precatheterisation chest radiograph influences the performance of the catheter procedure. They audited their routine adult catheterisation procedures (340 examinations) for 2 months. They asked the operators "Did the chest radiograph influence the choice of catheter?", "Did it influence the volume of contrast used?", and "Did it give any other useful information?"' Ischaemic (82\%) and valvar $(11 \%)$ heart disease predominated in their patients, with congenital heart disease accounting for only $3 \%$ of examinations and cardiomyopathy, pulmonary hypertension, and transplant assessment for the remainder.

The choice of catheter may be influenced by the anatomy in patients with congenital heart disease. In patients with coronary or valvar disease the size and shape of the aorta and presence of aortic valve calcification may make it difficult to cross the aortic valve and this may influence the choice of catheter or suggest that the chance of success would be improved if the brachial approach were use. However, the aortic contour and size can be estimated and valve calcification identified by fluoroscopy. Echocardiograms, which are likely to have been performed in all patients with valvar disease and many with coronary disease, give more accurate measurements than radiographic techniques. Even if an inappropriate catheter is used initially the cost savings from fewer chest radiographs will override this additional expense.

It is important to clarify the circumstances in which a "routine" chest radiograph is taken. The survey reported on page 540 considered diagnostic cardiac catheterisation: the situation is very different in patients undergoing angioplasty. Because some angioplasty patients will need bypass surgery a valid case can be made for a radiograph to be obtained before the procedure. Similarly, a patient admitted for diagnostic catheterisation who has experienced worsening clinical symptoms or signs may need a radiograph as part of the overall assessment. On the other hand, patients admitted for routine investigation either as a day case or for an overnight stay are a separate group. They are clinically stable and if routine examination shows no evidence of a significant deterioration in their condition a chest radiograph will not provide additional information. Indeed a recent radiograph will often be available.

The possibility that a small asymptomatic bronchial neoplasm will not be identified has to be considered. Such cancers are seldom found when radiographs are performed for other reasons ${ }^{1}$. The cost benefit of such screening is negligible. When a catheter procedure shows cardiac disease requiring intervention a radiograph will be obtained then anyway.

Stables and Trotman-Dickenson found that in patients with ischaemic heart disease the radiographs played no part in determining the appropriate catheter or the volume of contrast to be used. The choice of contrast agent and of its volume raises several issues. In patients with incipient or actual heart failure sodium overload should be avoided and a non-ionic contrast agent should be used. Such a decision, however, need not be based on a radiographic finding. The clinical history and examination should be sufficient to indicate the problem and a radiograph would then be clinically indicated. Echocardiography is a more useful examination for assessing left ventricular function and for examining patients with valvar disease. Because higher contrast volumes may be needed in patients with volume overload and lower contrast volumes in patients with outflow obstruction, the echocardiogram will provide more accurate data than a chest radiograph. Although pulmonary hypertension should alert the operator to the need to limit the contrast volume the necessity for this will be apparent from direct measurement or echocardiography.

The radiation dose to the patient from a chest radiograph is very small $(0.01-0.05 \mathrm{mSv})$. The International Committee for Radiological Protection recommends that a member of the public should not exceed an annual dose of $1 \mathrm{mSv}$ in the course of their daily life. Nevertheless, a review of the exposures associated with the explosion of the atomic bomb at Hiroshima indicates that the risk of cancer from exposure to ionising radiation is significantly higher than previously supposed. ${ }^{2}$ Any reduction in exposure to ionising radiation is to be commended.

A chest radiograph costs the National Health Service between $£ 13$ and $£ 15$. In a unit performing 2500 adult catheterisation procedures a year a conservative estimate suggests that at least half these procedures will be for the non-urgent assessment of ischaemic heart disease. If these patients did not have a chest radiograph $£ 16000-£ 19000$ a year would be saved. This would more than compensate for an occasional incorrect choice of initial catheter.

For the day cases or patients staying overnight with ischaemic or valvar heart disease who are clinically stable, a pre-catheter chest radiograph does not contribute to management of the procedure. A case can be made for routine precatheterisation radiographs in the small group of patients with adult congenital heart disease. If radiographs were not routinely performed in patients with ischaemic and valvar disease the radiation burden would be reduced and significant cost savings achieved.

Department of Imaging

MICHAEL PEARSON

Cardiac Department, Royal Hospitals Trust,

The London Chest Hospital, Bonner Road, London E2 9fX

1 Strauss GM, Gleason RE, Sugarbaker DJ. Screening for lung cancer reexamined A re-interpretation of the Mayo Lung Project randomized trial on lung cancer screening. Chest 1993; 103:337S-341S.

International Committee for Radiological Protection. Issue No. 60. (1990 recommendations). 